\title{
"Melting brain" as complication of a vein of galen aneurysmal malformation diagnosed by fetal MRI
}

\author{
Florian Hergan $^{1,2}$ and Thierry AGM Huisman ${ }^{1 *}$ \\ ${ }^{1}$ Section of Pediatric Neuroradiology, Division of Pediatric Radiology, Russell H. Morgan Department of Radiology and Radiological Science, The Johns Hopkins \\ University School of Medicine, Baltimore, MD, USA \\ ${ }^{2}$ Medical University of Graz, Austria
}

\begin{abstract}
The Vein of Galen aneurysmal malformation (VGAM) is a rare and complex arteriovenous (AV) malformation found in about $1 \%$ of all fetal AV abnormalities. The high morbidity and mortality requires an early and precise diagnosis to counsel parents and guide physicians for pre-, peri- and postnatal management as well as for prediction of outcome. Fetal MRI is a safe, important and well-established diagnostic tool in the clinical evaluation of fetuses with suspected cerebral anomalies and has become superior to color Doppler ultrasonography in the diagnosis of VGAM in recent years. It provides highly sensitive information not only about the VGAM and related brain injury but also about the systemic impact of the malformation on the fetus. In this report we present the fetal MR imaging findings including the progressing brain injury known as "melting brain".
\end{abstract}

\section{Introduction}

Arterio-venous anomalies involving the vein of Galen are rare and complex vascular lesions with an incidence of about one in 25000 with a male predominance of 3:1. According to Lasjaunias [1] these vascular lesions can be classified by its angioarchitectural characteristics as a VGAM, and a vein of Galen aneurysmal dilatation (VGAD). A VGAM is an arterio-venous (AV) malformation in which arteries directly connect to a persistent dilated embryonic prosencephalic vein of Markowski whereas in a VGAD arteries directly drain into a dilated but already formed vein of Galen. The vein of Galen is a short trunk which is being formed by the union of the two internal cerebral veins and the basal veins of Rosenthal. If left untreated a VGAM or VGAD has an almost $100 \%$ morbidity and mortality. The first attempts of treating these malformations were recorded in an infant with intracranial hypertension in the early $20^{\text {th }}$ century $[1,2-8]$. It can be diagnosed as early as week 6 to 11 of gestation, but is usually not detected prior to the $24^{\text {th }}$ week of gestation [9]. Two- (2D) and three- (3D) dimensional ultrasound (US), Doppler color US and magnetic resonance imaging (MRI) are the main diagnostic techniques during pregnancy.

Due to the significant arterio-venous shunting and "arterialization" of the draining vein of Galen or persistent vein of Markowski, the normal drainage of the intracerebral veins is impaired increasing the risk for chronic intraperenchymal venous stasis and/or ischemia. This chronic venous congestion may result in a progressive destruction of the hemispheric white matter and overlying cortex known as the "melting brain syndrome". Furthermore the increased venous pressure impairs the resorption of cerebrospinal fluid at the Pachionic granulations with subsequent hydrocephalus which may be aggrevated by direct compression and obstruction of the Sylvian aqueduct by the dilated vein of Galen $[3,6]$. Furthermore systemic complications of the AV-shunting include congestive heart failure, pleural and pericardial effusions, ascites as well as generalized subcutaneous edema (fetal hydrops).
As described in the literature $[7,10]$ VGAM/VGAD have a very poor outcome, in particular if a "melting brain" develops or significant systemic complications ensue. Termination of the pregnancy is often considered when the fetus presents with multi-organ failure or brain parenchymal change. However, there are certain factors like Neonatal score $>12$ (described by Lasjaunias) or no parenchymal changes $(\mathrm{p}<0.003)$, no calcifications $(\mathrm{p}<0.046)$, or no arterial steal phenomenon $(\mathrm{p}<0.001)$ which are statistically relevant and predict a better patient outcome and possible survival [10]. An early and complete diagnosis during fetal life is consequently essential.

We report on two different cases with fetal melting brain syndrome to emphasize the ability of fetal MRI to detect malformation and its cerebral and systemic complications.

\section{Case report}

In a 29 year old pregnant (P0121) woman, a sonogram showed intracranial lateral ventricle dilation of the fetus at 25 weeks of gestation. The corpus callosum appeared slightly wider than expected. A fetal MRI (Figure 1) was done showing moderate contour irregularity along both lateral ventricles with inhomogeneous T2 hypointense signal of the periventricular white matter along both lateral ventricles. Moderate-to-severe supratentorial hydrocephalus and volume loss of the periventricular white matter consistent with a developing "melting

Correspondence to: Thierry AGM Huisman, MD, Professor of Radiology, Pediatrics, Neurosurgery and Neurology. Director Division of Pediatric Radiology and Pediatric Neuroradiology, The Russell H. Morgan Department of Radiology and Radiological Science, The Johns Hopkins School of Medicine, Charlotte R. Bloomberg Children's Center, Sheikh Zayed Tower, Room 4174, 1800 Orleans Street, Baltimore, MD 21287-0842, USA, Tel: +14109556454; Fax: +14105023633; E-mail: thuisma1@jhmi.edu

Received: November 21, 2017; Accepted: December 11, 2017; Published: December 15, 2017 


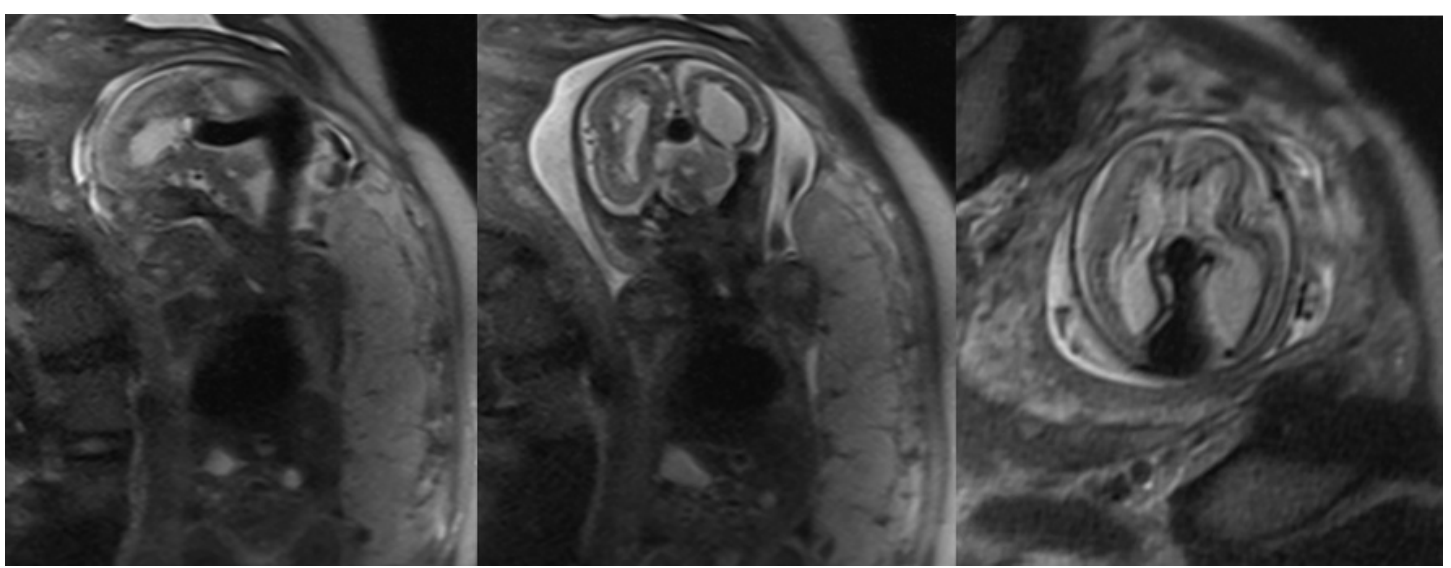

Figure 1. Triplanar (sagittal, coronal and axial) T2-weighted ultrafast fetal MRI in a fetus with a VGAM. The vein of Galen is seen as an enlarged, T2-hypointense tubular structure in the midline draining into the dilated Torcula Herophilli and subsequent transverse and sigmoid sinuses. The periventricular white matter is significantly reduced in volume and mixed T2-hyper and hypo-intense, the ventricles are enlarged. Findings are characteristic for a melting brain syndrome secondary to a VGAM.

brain" as well as moderate cardiomegaly was detected. A significantly dilated vein of Galen (Figure 1) as well as enlargement of the straight sinus, transverse sinuses and sigmoid sinuses was seen. The posterior cereberal arteries and posterior choroidal arteries were also dilated. A follow up ultrasound examination at 31 weeks gestation showed a worsening fetal hydrops, cardiomegaly, ascites, hypoplastic aortic arch with accelerated flow, bilateral cerebral ventriculomegaly, hydrocele. A fetal echocardiogram confirmed the cardiomegaly with a dilated rightsided heart including markedly dilated superior vena cava consistent with a history of a vein of Galen Malformation. A moderate tricuspid regurgitation and moderately depressed right ventricular systolic function was also found.

The mother was admitted for prolonged fetal monitoring, 2 doses of betamethasone, and C-section 24 hours after the last steroid dose. 36 hours after administration of her first dose of bethamethasone her Biophysical Profile became increasingly concerning (4/10) prior to $6 / 10$ at the time of admission and the decision was made to proceed with a delivery via C-section prior to the planned 48 hours post betamethasone time. The mother tolerated the procedure well, the baby went to the NICU but did not survive its first day of life.

\section{Case 2}

In a 29 year old (P0G1) woman, routine 14-week ultrasound showed a large anterior uterine fibroid which was confirmed on a follow up ultrasound study done at 21 weeks. The fetal anatomy at that time was reported normal. The 25-week ultrasound study was concerning for an enlarged right $>$ left fetal heart. After presenting with vaginal bleeding a maternal fetal medicine ultrasound study was performed which showed a large midline "cystic" structure with dilated intracranial vessels including a large superior sagittal sinus. There were multiple arteries near the "cystic" structure. Pulse Doppler showed high velocity flow profiles consistent with a fetal vein of Galen aneurysmal malformation. Right sided cardiomegaly was seen with moderate-tosevere systolic tricuspid regurgitation. Enlarged vena cava and dilated neck veins were also reported.

In order to further evaluate the anomaly a fetal MRI (Figure 2a) was performed which confirmed a large T2 hypointense tubular structure in the region of the expected location of the vein of Galen, significant elongation and partially deviation to the right of the midline of the vein of Galen which was draining into a significantly dilated torcula Herophilli as well as significant dilatation/widening of the transverse sinus bilaterally, sigmoid sinus and jugular veins. Multiple dilated feeding arteries were noted originating from the circle of Willis predominantly of the carotid arteries bilaterally, draining into the dilated vein of Galen to the right of the midline. The ventricles were of normal size for gestational age, the hemispheric white matter and cortical gray matter unremarkable. A follow up fetal MRI 4 weeks (Figure 2b) later confirmed the vein of Galen aneurysmal malformation, however the periventricular white matter appeared significantly injured with rapidly progressing resorption of the periventricular white matter, intraparenchymal hemorrhages/calcifications, malformed overlying cortical ribbon and a vague dilatation of the ventricles consistent with a "melting brain" syndrome.

At 31 weeks the child was delivered via primary low-segment transverse cesarean section. The mother tolerated to procedure well and the baby was brought to the NICU where it passed away after 27 minutes of life.

\section{Discussion}

The Vein of Galen Aneurysmal Malformation is a rare vascular malformation with an incidence of $1 \%$ of all fetal $\mathrm{AV}$ abnormalities $[1,8,9]$. Morbidity and mortality is high, especially if a progressive brain injury develops in combination with systemic symptoms. Even though the vascular anomaly is being formed between the $6^{\text {th }}$ and $11^{\text {th }}$ week of gestation it usually remains un-detected until the end of the second trimester. Color coded Doppler ultrasonography typically allows to identify the vascular anomaly, however progressing white matter injury may remain underrecognized. Fetal MRI is a safe, important and well-established diagnostic tool in the clinical evaluation of fetuses with suspected cerebral anomalies and has become a valuable adjunct to color Doppler ultrasonography in the diagnosis of VGAM/VGAD in recent years [11-13]. Not only can the MRI detect cerebral anomalies in more detail but it can also give a highly sensitive information about the brain ultrastructure as well as associated systemic complications including fetal cardiac failure, pleural effusions, ascites, fetal hydrops and even placental hydrops. The "melting brain" syndrome is caused by chronic hypoxia and venous stasis due to venous hypertension which leads to rapid destruction of the white matter $[1,11]$. The presence of these factors are associated with poor postnatal outcome for the fetus and may result in maternal complications if not detected early. Fetal MRI provides highly sensitive imaging information relevant for prognostication and management of pregnancy and delivery. Serial or follow up fetal MRI may be necessary to identify progressive brain 

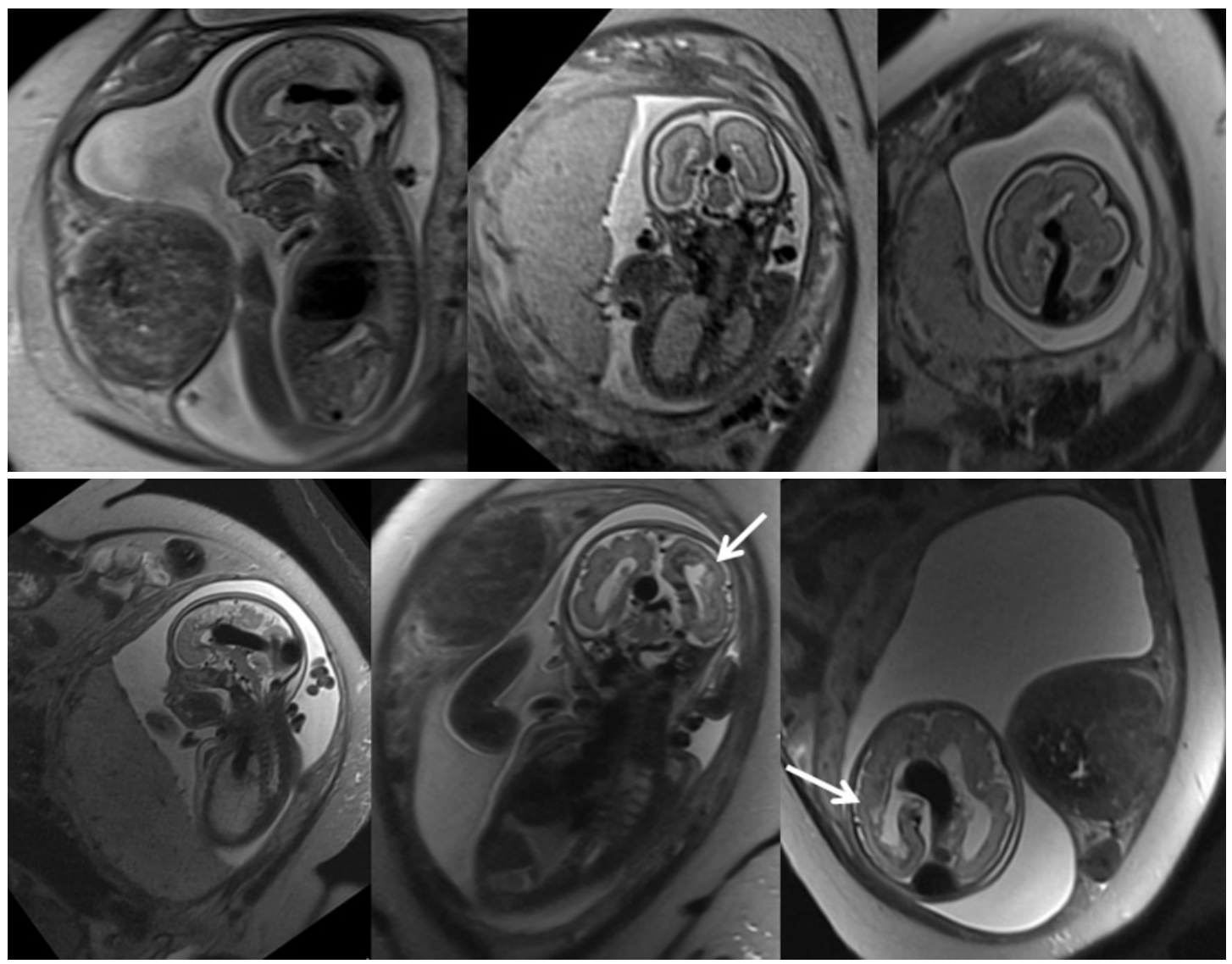

Figure 2. Triplanar (sagittal, coronal and axial) T2-weighted ultrafast fetal MRI in a fetus with a VGAM. The initial fetal MRI (a) shows the characteristic dilated vein of Galen draining into a dilated torcula Herophilli and transverse sinus. The periventricular white matter and overlying cortical ribbon are appropriate for gestational age. The follow up fetal MRI (b) four weeks later shows progressive periventricular white matter injury with developing ventriculomegaly and a malformed overlying cortical ribbon. Mild subcutaneous edema is noted along the chest wall. On both studies a large uterine fibroid is noted.

injury which may be too subtle or even absent on an initial, early study. Radiologists should be familiar with this devastating and characteristic "melting brain" imaging findings.

\section{References}

1. Lasjaunias P, Brugge K ter, Rodesch G, Burrows P, Piske R, et al. (1997) Vascular Diseases in Neonates, Infants and Children. Berlin Heidelberg: Springer Verlag Berlin Heidelberg.

2. Hrishi AP, Lionel KR (2017) Periprocedural Management of Vein of Galen Aneurysmal Malformation Patients: An 11-Year Experience. Anesth essays Res 11: 630-635. [Crossref]

3. Tortori-Donati P, Rossi A, Biancheri R (2005) Pediatric Neuroradiology Brain. Berlin Heidelberg: Springer Berlin Heidelberg.

4. Bhattacharya JJ, Thammaroj J (2017) Vein of galen malformations. J Neurol Neurosurg Psychiatry 74 Suppl 1(suppl 1): i42-i44. [Crossref]

5. Stephan S, Rodesch G, Elolf E, Wiemann D, Jorch G (2012) Vein of galen aneurysmal malformations: an ultrasonographic incidental finding-a case report. Case Rep Pediatr 2012: 824-284. [Crossref]

6. Puvabanditsin S, Mehta R, Palomares K, Gengel N, Da Silva CF, et al. (2017) Vein of Galen malformation in a neonate: A case report and review of endovascular management. World J Clin Pediatr 6: 103-109. [Crossref ]

7. Herghelegiu D, Ionescu CA, Pacu I, Bohiltea R, Herghelegiu C, et al. (2017) Antenatal diagnosis and prognostic factors of aneurysmal malformation of the vein of Galen: A case report and literature review. Medicine (Baltimore) 96: e7483. [Crossref]

8. Madhuban A, van den Heuvel F, van Stuijvenberg M (2016) Vein of Galen Aneurysmal Malformation in Neonates Presenting With Congestive Heart Failure. Child Neurol Open 3: 2329048X15624704. [Crossref]
9. Bohiltea RE, Turcan N, Mihalea C, Dorobat B, Cinteza EE, et al. (2016) Ultrasound Prenatal Diagnosis and Emergency Interventional Radiologic Therapy of Galen Aneurysmal Malformation in a Newborn. Maedica (Buchar) 11: 334-340. [Crossref]

10. Geibprasert S, Krings T, Armstrong D, terBrugge KG, Raybaud CA (2017) Predicting factors for the follow-up outcome and management decisions in vein of Galen aneurysmal malformations. Child's Nerv Syst 26: 35-46. [Crossref]

11. Wagner MW, Vaught AJ, Poretti A, Blakemore KJ, Huisman TAGM (2015) Vein of galen aneurysmal malformation: prognostic markers depicted on fetal MRI. Neuroradiol J 28: 72-75. [Crossref]

12. Komiyama M, Nakajima H, Nishikawa M, Yamanaka K, Iwai Y, et al. (2001) Vein of galen aneurysms. Experience with eleven cases. Interv Neuroradiol 7(Suppl 1): 99103. [Crossref]

13. Zhou LX, Dong SZ, Zhang MF (2017) Diagnosis of Vein of Galen aneurysmal malformation using fetal MRI. J Magn Reson Imaging 46: 1535-1539. [Crossref]

Copyright: (C2017 Hergan F. This is an open-access article distributed under the terms of the Creative Commons Attribution License, which permits unrestricted use, distribution, and reproduction in any medium, provided the original author and source are credited. 